\title{
O IMPACTO DA ADOÇÃO NO DESENVOLVIMENTO DA CRIANÇA ${ }^{1}$
}

\author{
Joana Baptista ${ }^{2}$ \\ Isabel Soares ${ }^{3}$ \\ Margarida Henriques ${ }^{2}$
}

[Enviado a 28-11-2011. Aceite a 24-09-2012]

Resumo: Em comparação com os pares da comunidade, as crianças que foram adotadas enfrentam, com maior frequência, adversidade e risco nos primeiros tempos de vida, os quais se podem situar ao nível de complicações ao nascimento e de experiências de perda da família biológica, bem como ao nível do acolhimento em instituições caracterizadas por cuidados pouco responsivos. Nos últimos anos, clínicos e investigadores têm vindo a alertar para os efeitos negativos daquelas experiências no desenvolvimento da criança. Simultaneamente, a literatura tem vindo a reforçar a ocorrência de mudanças positivas em áreas diversas do desenvolvimento, após a integração numa família por adoção. Este artigo de revisão apresenta uma sistematização de resultados de estudos que analisaram o impacto da adoção, sobretudo internacional, no desenvolvimento físico, cognitivo, sócio-emocional e nos problemas de comportamento da criança que foi adotada.

Palavras-chave: adoção; crescimento; desenvolvimento; vinculação.

The impact of adoption on child's development (Abstract): Compared with their peers, adopted children face, more often, adversity and risk in early life, namely in terms of complications at birth, loss of the biological family and the admission in institutional settings characterized by less responsive care. Over the past few

\footnotetext{
${ }^{1}$ Este artigo foi redigido com o apoio da Fundação Bial - Bolsa 13/06 e da Fundação para a Ciência e Tecnologia - PTDC/PSI - PCL/101506/2008

2 Faculdade de Psicologia e de Ciências da Educação da Universidade do Port. Endereço para correspondência:

Faculdade de Psicologia e de Ciências da Educação da Universidade do Porto, Rua Alfredo Allen, 4200-135 Porto PORTUGAL. E-mail: joanabaptist@gmail.com

${ }^{3}$ Escola de Psicologia da Universidade do Minho
} 
years, clinicians and researchers have warned about the negative effects of those experiences in child development. Simultaneously, the literature has been reinforcing the occurrence of positive changes after adoption in several areas of development. This paper review presents a summary of the main empirical findings about the effects of adoption, especially international, in child's physical growth, cognitive, socio-emotional development and behavioral problems in adopted children.

Keyword: adoption; growth; development; attachment

\section{Introdução}

Com a queda do regime de Nicolae Ceauşescu na Roménia, em 1989, tornaram-se públicas as condições e as experiências de privação severa a que as crianças institucionalizadas naquele país estavam expostas, muitas desde a primeira infância. Esta situação, altamente preocupante e atípica do ponto de vista da adversidade extrema encontrada, conduziu a uma resposta humanitária que envolveu a adoção internacional de muitas daquelas crianças, por parte de famílias da Europa Ocidental e da América do Norte (Rutter et al., 2009). Do ponto de vista teórico e científico, esta circunstância abriu portas para a realização de experiências naturais (Haugaard \& Hazan, 2003), na procura de respostas à seguinte questão: até que ponto é possível a recuperação, em diferentes domínios do desenvolvimento, em crianças que foram expostas a experiências precoces de privação de cuidados parentais adequados, após a sua integração num contexto de cuidados de maior qualidade?

Com o intuito de analisar o impacto da exposição precoce a condições de cuidados desfavoráveis, investigadores têm dedicado, nos últimos anos, uma atenção crescente ao estudo dos resultados e dos processos desenvolvimentais em crianças que foram adotadas internacionalmente (Palacios \& Brodzinsky, 2010). Apesar das experiências adversas vividas no período que antecedeu a adoção - e.g., complicações ao nascimento, negligência e/ou maus-tratos vivenciados na família biológica, cuidados em contexto institucional pouco individualizados -, vários estudos têm revelado ganhos acentuados em diferentes áreas do desenvolvimento após a integração da criança na família por adoção (Cohen, Lojkasek, Zadeh, Pugliese, \& Kiefer, 2008; Johnson, 2000; Juffer \& Van IJzendoorn, 2009; Londen, Juffer, \& Van IJzendoorn, 2007; Tan \& Yang, 2005; Van IJzendoorn, Juffer, \& Klein Poelhuis, 2005). Simultaneamente, estudos levados a cabo com crianças que foram adotadas têm permitido identificar a presença de variações individuais, quer no grau em que o desenvolvimento da criança que foi adotada é afetado pelas experiências anteriores, quer no nível de recuperação posterior 
(Rutter et al., 2009; Rutter, Kreppner, O’Connor, \& The ERA Study Team, 2001).

De seguida, são apresentados os resultados de estudos que se debruçaram sobre a análise do desenvolvimento da criança que foi adotada, sobretudo internacionalmente, alguns dos quais realçam o seu estatuto, quer no momento de chegada à família por adoção, quer nos meses e anos seguintes à adoção. A presente revisão da literatura foi operacionalizada em duas fases. Durante a primeira fase deu-se início à pesquisa em duas plataformas eletrónicas de base de dados (i.e., EBSCOhost e ISI Web of Knowledge) e as consultas incluíram o período entre 1945 e 2011 . Foram utilizados como descritores os seguintes termos: adopção (adoption), adoptado (adoptee), recuperação desenvolvimental após a adopção (developmental recovery after adoption), crianças pós-institucionalizadas (post-institutionalized children), crianças institucionalizadas (institution-reared children) e institucionalização (institutionalization). A segunda fase desta revisão foi dedicada à exclusão dos trabalhos não relevantes à pesquisa, primeiramente através de uma análise do resumo e, em seguida, através de uma avaliação mais detalhada, com base na leitura integral e na identificação dos principais resultados. Do total, foram encontradas 150 produções possivelmente relevantes e 57 foram selecionadas após a avaliação detalhada (i.e., 43 estudos originais, quatro estudos de revisão e cinco capítulos de livros, dois relatórios e três comunicações apresentadas em congressos internacionais). No que concerne aos critérios de inclusão, foram identificados estudos, desde clássicos a atuais, que se debruçaram sobre a análise do impacto da adoção no desenvolvimento da criança, nos seguintes domínios: (a) desenvolvimento físico, (b) desenvolvimento cognitivo, (c) desenvolvimento sócio-emocional, e (d) problemas de comportamento.

\section{Desenvolvimento fisico}

São vários os estudos que têm documentado os efeitos negativos da institucionalização no desenvolvimento físico da criança (e.g., Frank, Klass, Earls, \& Eisenberg, 1996; Johnson, 2000). Na verdade, este é o problema mais frequentemente observado em crianças institucionalizadas (Bakwin, 1949; Fried \& Mayer, 1948; Smyke, Koga, Johnson, Zeanah, \& The BEIP Core Group, 2004) e recentemente adotadas (e.g., Le Mare \& Audet, 2006; Loman, Wiik, Frenn, Pollack, \& Gunnar, 2009; Miller, Chan, Comfort, \& Tirella, 2005; Pormerleau et al., 2005). Porém, quando a criança é retirada de um contexto de privação de cuidados, verifica-se uma acentuada e imediata recuperação (Johnson, 2000, 2002). Estudos de follow-up com crianças que foram adotadas internacionalmente têm confirmado este padrão de mudança (e.g., Ames, 1997; Rutter \& The ERA Study Team, 1998), havendo evidência (e.g., Gunnar, 2000; Johnson, 2000) de que crianças recentemente adota- 
das tendem a apresentar ao nível do comprimento, peso e perímetro cefálico (entendido como uma medida indireta do crescimento cerebral) valores inferiores aos apresentados por crianças da mesma idade integradas na família biológica. Nos meses seguintes à chegada à família por adoção, verifica-se a ocorrência de mudanças significativas ao nível do crescimento, naqueles três parâmetros.

Num estudo conduzido no Reino Unido, que monitorizou o desenvolvimento físico de crianças romenas desde o momento da sua integração na família por adoção até aos 11 anos de idade, os autores observaram ganhos ao nível do desenvolvimento físico, e verificaram que uma idade precoce no momento da adoção contribui grandemente para os mesmos (O'Connor, Rutter \& The ERA Study Team, 2000; Rutter \& The ERA Study Team, 1998). Os autores constataram que $51 \%, 34 \%$ e $38 \%$ das crianças no momento da adoção apresentavam um comprimento, peso e perímetro cefálico, respetivamente, abaixo do percentil três, mas aos quatro anos de idade aquelas percentagens baixaram para $1 \%, 2 \%$ e $13 \%$. A recuperação neste domínio foi notória, tendo-se mantido até aos seis anos de idade. Porém, na reavaliação levada a cabo aos seis anos de idade, concluiu-se que as crianças que foram adotadas mais tardiamente tinham menos peso e um menor perímetro cefálico, do que as crianças romenas adotadas mais cedo. Independentemente da idade, o perímetro cefálico revelou-se como a única medida cujos valores, aos quatro, seis e 11 anos de idade, ficavam aquém do esperado (Rutter et al., 2009).

Estes resultados que revelam o padrão de ganhos no peso e no comprimento após adoção, bem como no perímetro cefálico apesar de mais lento, foram confirmados por uma meta-análise (Van IJzendoorn, Bakermans-Kranenburg, \& Juffer, 2007), na qual se constatou, 8 anos depois da integração na família por adoção, ganhos consideráveis ao nível do comprimento e do peso ( 23 e 18 estudos; 3.437 e 3.259 crianças, respetivamente). Os autores verificaram que, no momento da integração na família por adoção, a força da diferença ao nível do comprimento entre as crianças que foram adotadas e as crianças da comunidade era de $d=-2.43^{4}$, sendo independente da idade. Todavia, anos após a integração na família por adoção, as crianças continuavam a ser mais baixas do que os pares da comunidade, pelo que, segundo os autores, a puberdade poderá ser uma das causas explicativas do facto da estatura final ficar aquém daquilo que seria de esperar, tendo em conta a rápida e acentuada recuperação verificada. No entanto, é de salientar que, anos após a adoção, aquela diferença é consideravelmente menor, em

${ }^{4}$ De acordo com Cohen (1988), um $d$ até 0.20 representa um tamanho de efeito pequeno, um $d$ de 0.50 um tamanho de efeito moderado, e um $d$ igual ou superior a 0.80 um tamanho de efeito acentuado. 
comparação com a diferença registada no momento da adoção. O mesmo padrão de ganhos foi identificado para o peso. Quanto ao perímetro cefálico, observou-se um processo mais lento de mudança, sugerindo que o crescimento cerebral parece ser mais suscetível do que o comprimento e o peso a efeitos negativos duradouros.

Apesar do prognóstico favorável ao nível do desenvolvimento físico, alguns estudos (e.g., Teilmann, Pedersen, Skakkebaek, \& Jensen, 2006) têm alertado para o fato das crianças que foram adotadas internacionalmente e do sexo feminino constituírem um grupo de risco para a ocorrência precoce da puberdade, o que poderá levar a uma menor estatura. Tuvemo e Proos (1993), por exemplo, concluíram que $13 \%$ das crianças que foram adotadas por famílias na Suécia, e provenientes da Índia, atingiram a menarca antes dos 10 anos de idade, sendo o risco maior nas crianças com um menor comprimento no momento de integração na família por adoção, mas com uma recuperação mais acentuada. A probabilidade de ocorrência precoce da puberdade parece ser maior quanto mais tardia for a idade na adoção (Teilman et al., 2006). Apesar da etiologia da menarca precoce não ser ainda totalmente compreendida, aqueles autores sugerem que a mesma poderá ser explicada por fatores diversos, nomeadamente (1) a acumulação e a durabilidade de acontecimentos de vida stressantes, durante a primeira e a segunda infância, associados a uma idade mais tardia na adoção; e (2) a presença de determinados estímulos ambientais na família por adoção (e.g., melhoria das condições nutricionais e um padrão de crescimento instável), que exercem influência no sistema límbico e que conduzem a alterações metabólicas e endócrinas (e.g., alterações nos fatores de crescimento semelhantes à insulina ou somatomedinas). A puberdade precoce, por sua vez, poderá levar à fusão prematura da cartilagem de crescimento, reduzindo a duração do crescimento e a estatura final.

\section{Desenvolvimento Cognitivo}

O desenvolvimento cognitivo de crianças institucionalizadas e que foram adotadas é alvo de estudo há mais de 60 anos. Entre 1930 e 1950 surge o primeiro conjunto de estudos, a partir do qual são documentados défices cognitivos e atrasos na linguagem, sendo que resultados semelhantes foram encontrados em estudos posteriores, uns menos recentes (e.g., Bowlby, 1951; Provence \& Lipton, 1962; Spitz, 1945) outros mais atuais (e.g., Loman et al., 2009; Londen et al., 2007; Zeanah, Smyke, Koga, Carlson, \& The Beip Core Group, 2005), havendo evidência de que no momento da adoção as crianças que estiveram previamente em instituições tendem a evidenciar atrasos no desenvolvimento cognitivo (Miller \& Hendric, 2002), seguindo-se, meses depois, a ocorrência de ganhos consideráveis (Rutter, O'Connor \& The ERA Study Team, 2004). 
Tal como para o desenvolvimento físico, diferentes estudos têm apontado para uma recuperação massiva ao nível do desenvolvimento cognitivo, após a integração da criança na família por adoção. Num estudo com 123 crianças que foram adotadas antes dos 18 meses de idade, Pormerleau et al. (2005) avaliaram o desenvolvimento cognitivo das crianças antes de completarem um mês de integração na família por adoção, e três e seis meses depois. Entre a avaliação inicial e os seis meses verificou-se uma evolução positiva ao nível do desenvolvimento cognitivo, apesar de os resultados permanecerem abaixo do que seria de esperar para a idade, tal como mais recentemente foi evidenciado por outro estudo (i.e., Van den Dries, Juffer, Van IJzendoorn, \& Bakermans-Kranenburg, 2010). Estes resultados vão assim ao encontro do estudo de Cohen et al. (2008), no qual se concluiu que a completa recuperação no domínio cognitivo não ocorre antes dos dois anos de integração na família por adoção. Cohen et al. (2008) examinaram as trajetórias de desenvolvimento de 70 crianças do sexo feminino provenientes da China e que foram adotadas por famílias Canadianas. As crianças foram avaliadas no momento da adoção, com 13 meses de idade, e reavaliadas seis, 12 e 24 meses depois. Quarenta e três crianças da comunidade, da mesma idade e género, serviram de grupo de controlo. Os autores concluíram que na avaliação inicial as crianças que foram adotadas apresentavam desempenhos significativamente inferiores aos das crianças da comunidade em termos cognitivos, sendo que o mesmo já não se verificou na reavaliação dos 24 meses após a adoção.

Será que as crianças que foram adotadas tendem a apresentar resultados mais positivos em termos do seu desenvolvimento cognitivo do que as crianças que permaneceram institucionalizadas? A este respeito, numa série de meta-análises concluiu-se que as crianças que foram adotadas apresentavam um desempenho significativamente superior ao das crianças que permaneceram em instituições em termos de Quociente de Inteligência (Q.I.). Todavia, quando comparadas com os pares da comunidade, as crianças que foram adotadas parecem não diferir em termos de Q.I., no que concerne à adoção internacional. Parecem apresentar, porém, um atraso, apesar de ligeiro, em termos de linguagem, quando comparadas com os pares da comunidade (Van IJzendoorn et al., 2005), bem como, tal como evidenciado noutros estudos, piores desempenhos em provas de memória (Bauer, Hanson, Pierson, Davidson, \& Pollak, 2009; Pollak et al., 2010) e funções executivas (Bauer et al., 2009).

\section{Desenvolvimento Sócio-Emocional}

A investigação tem vindo a documentar de forma consistente a ocorrência de dificuldades sócio-emocionais em crianças institucionalizadas (Zeanah et al., 2009). Porém, a questão que se coloca é se estes problemas 
persistem após a adoção. Aliado a isto, estarão as crianças que foram adotadas em maior risco de evidenciar padrões específicos de problemas emocionais e sociais?

Diferentes estudos apontam para a ocorrência de comportamentos atípicos em crianças que foram adotadas e cuja história de cuidados anterior à adoção é marcada pela vivência em acolhimento institucional. Assim, estereotipias (i.e., repetição automática dos movimentos, como o balançar do corpo) foram observadas em mais de $50 \%$ das crianças da Europa do Leste que foram adotadas por famílias canadianas (Benoit, Joycelyn, Moddemann, \& Embree, 1996). Resultados semelhantes têm sido encontrados noutros estudos. Também Beckett et al. (2002) encontraram uma percentagem elevada de crianças que apresentavam estereotipias (47\%), bem como comportamentos de autoagressão (24\%), no momento da adoção. Aos seis anos de idade, os autores constataram que aquelas percentagens tinham diminuído consideravelmente (18\% e 13\%, respetivamente), pelo que verificaram ainda dificuldades no relacionamento com os pares.

O estudo ERA veio chamar a atenção para a presença de um padrão de comportamento do tipo autístico em algumas crianças romenas que foram adotados por famílias do Reino Unido, marcado por dificuldades ao nível da reciprocidade social e comunicação e pela presença de uma preocupação excessiva por interesses particulares (Kumsta et al., 2010), devido à vivência de experiências prévias relacionais inadequadas (Rutter et al., 2009). Os autores concluíram que $6 \%$ de uma amostra de 111 crianças apresentava aquele padrão, pelo que, aos quatro anos de idade, o padrão era indistinguível do autismo "típico". Todavia, aos seis anos de idade registou-se uma acentuada diminuição das características autisticas, acompanhada de uma maior flexibilidade na tentativa do uso de diferentes formas de comunicação (Kumsta et al., 2010). Aos 11 anos de idade, cerca de 1/4 das crianças deixaram de apresentar aquele padrão comportamental (Rutter et al., 2009).

No que concerne à (des)organização e aos comportamentos perturbados de vinculação, para a criança que foi adotada, a construção de uma relação de vinculação segura com a família por adoção é uma tarefa desafiante, considerando, por um lado, as experiências prévias de separação e de perda (Bowlby, 1982), e, por outro, as condições de privação social em contexto institucional, que podem dificultar a construção de uma relação seletiva e diferenciada com os pais por adoção (Zeanah et al., 2005).

\section{a) (Des)organização da Vinculação}

No âmbito de um projeto longitudinal (Juffer \& Rosenboom, 1997), que incluiu 160 crianças que foram adotadas antes dos seis meses de idade, procedeu-se à avaliação da qualidade da relação de vinculação entre a criança e os seus pais por adoção, após uma intervenção breve centrada no refor- 
ço dos comportamentos sensíveis maternos. Verificou-se que $74 \%$ das crianças eram seguras, o que é comparável com os valores encontrados na comunidade (i.e., 65\%; Van IJzendoorn \& Kroonenberg, 1988). Num outro estudo, $22 \%$ das crianças que foram adotadas foram classificadas como desorganizadas (Juffer, Bakermans-Kranenburg, \& Van IJzendoorn, 2005). Esta percentagem também não se diferenciava dos $15 \%$ identificados em populações normativas (Van IJzendoorn, Schuengel, \& Bakermans-Kranenburg, 1999). Contudo, Cohen e Farnia (2011) demonstraram que, apesar de a maioria das crianças adotadas da sua amostra apresentar uma vinculação segura, as mesmas tendiam a ser mais desorganizadas quando comparadas com as da comunidade.

Numa meta-análise, verificou-se que crianças que foram adotadas tendiam a apresentar resultados menos favoráveis do ponto de vista da (des)organização (i.e., $47 \%$ de crianças seguras e $31 \%$ de crianças desorganizadas), quando comparadas com pares da comunidade (i.e., $62 \%$ de crianças seguras e $15 \%$ de crianças desorganizadas). Todavia, observou-se também que aquelas crianças que foram adotadas apresentavam resultados mais favoráveis quando comparadas com crianças que permaneceram institucionalizadas (i.e., $11 \%$ de crianças seguras e $73 \%$ de crianças desorganizadas) (Van den Dries, Juffer, Van IJzendoorn, \& Bakermans-Kranenburg, 2009). Ademais, as crianças que foram adotadas antes dos 12 meses de idade não se diferenciavam dos pares da comunidade, relativamente à segurança da vinculação. Quanto à desorganização, não foram verificadas diferenças significativas em relação à idade na adoção. Sendo assim, estas meta-análises indicam que a adoção parece constituir-se, simultaneamente, como um fator de risco, resultante num maior número de relações de vinculação inseguras e desorganizadas (comparativamente com pares da comunidade), e como um fator protetor, capaz de levar à mudança (comparativamente com crianças institucionalizadas; e.g., Zeanah et al., 2005; 65\% de crianças classificadas como desorganizadas, respetivamente, neste estudo).

\section{b) Comportamentos Perturbados de Vinculação}

Um dos resultados mais frequentemente associado à experiência institucional e à privação de cuidados parentais tem sido a ocorrência de comportamentos indiscriminados, associados a défices noutros domínios psicológicos (Rutter et al., 2009), e que a investigação tem vindo a descrever como um problema clinicamente relevante com tendência a persistir anos após a adoção (O'Connor \& Zeanah, 2003), no caso das crianças que foram expostas a cuidados institucionais (O'Connor \& Spagnola, 2009; O'Connor et al., 2000; Rutter et al., 2007). 
Os sistemas de nosologia amplamente utilizados no âmbito das perturbações psiquiátricas (i.e., DSM-IV-TR, American Psychiatric Association, 2000; e ICD-10, World Health Organization, 2007) descrevem a Perturbação Reativa de Vinculação como um problema com início antes dos cinco anos de idade, que se caracteriza por relações sociais acentuadamente perturbadas na maior parte dos contextos e que parece estar associada a uma história de cuidados patogénicos, que se manifestam: (a) pela negligência permanente das necessidades emocionais básicas da criança e relacionadas com o conforto, estimulação e afeto; (b) pela negligência permanente das necessidades físicas básicas da criança; e (c) por mudanças repetidas da pessoa que trata primariamente da criança, o que impede a formação de vínculos estáveis. Esta perturbação pode manifestar-se de forma predominantemente inibida (i.e., dificuldade persistente para iniciar e responder à maioria das interações sociais de modo adequado, tendo em conta o nível de desenvolvimento da criança; retração e hipervigilância; constrição emocional e procura de proximidade do cuidador de forma bizarra ou ambivalente; a criança não se deixa confortar nem recorre ao cuidador em momentos mais exigentes) ou desinibida (i.e., vínculos difusos e sociabilidade indiscriminada; incapacidade para estabelecer vínculos seletivos adequados, uma vez que a criança procura contacto e proximidade com qualquer cuidador disponível, ou seja, de forma indiscriminada).

A literatura tem dado maior atenção ao tipo desinibido, visto que este parece ser mais comum do que o tipo inibido. Tendo por base os pressupostos da Teoria da Vinculação (Bowlby, 1982), o comportamento indiscriminado representa um desvio da organização normativa do sistema comportamental de vinculação. Os fatores etiológicos envolvidos estão longe de serem entendidos, apesar de consistentemente serem atribuídos a uma história de cuidados patogénica, tendo-se hipotetizado que o tipo desinibido da Perturbação Reativa de Vinculação poderá ser independente de uma relação de vinculação estabelecida (O’Connor \& Zeanah, 2003).

A este respeito, o estudo de Rutter et al. (2007) demonstrou que cerca de $71 / 2$ anos depois da adoção, $26 \%$ das crianças romenas que foram adotadas entre os 6-42 meses de idade, e que estiveram em instituições, apresentavam comportamentos indiscriminados de vinculação. Contrariamente, apenas $9 \%$ das crianças que foram adotadas e que não tinham experienciado cuidados institucionais, ou que foram integradas na família por adoção antes dos seis meses de idade, revelaram tais comportamentos. Estes resultados vão ao encontro dos identificados noutros estudos (e.g., Wallin, Dozier, Bick, \& Bernard, 2011), nos quais se constatou que os comportamentos indiscriminados de vinculação tendem a persistir anos após a integração da criança na família por adoção. 


\section{Problemas de Comportamento}

Num estudo canadiano, constatou-se que as crianças filhas por adoção apresentam mais problemas de externalização e internalização, comparativamente com pares da comunidade (Marcovitch et al., 1997). Os resultados do estudo de Marcovitch et al. (1997) vão ao encontro do recente estudo de Wiik et al. (2011), no qual se constatou a ocorrência de mais problemas de comportamento em crianças que foram adotadas e que tinham estado institucionalizadas. Merz e Mcall (2010), por sua vez, analisaram a ocorrência de problemas de comportamento em crianças que foram adotadas e que eram provenientes de instituições romenas, caracterizadas por (a) providenciarem cuidados de saúde e de nutrição adequados, mas (b) por apresentarem simultaneamente mudanças frequentes de cuidadores, escassas oportunidades de interação entre a criança e o adulto e cuidados pouco responsivos. Concluíram que as crianças provenientes de instituições revelavam mais problemas de externalização e de atenção do que os pares nunca institucionalizados e que sempre viveram com a família biológica.

Não obstante, outros estudos revelam que a maioria das crianças que foram adotadas encontra-se bem adaptada, anos depois da integração na família por adoção (Palacios \& Sánchez-Sandoval, 2005; Rutter et al., 2001). Assim sendo, alguns estudos apresentam resultados divergentes dos anteriores, quanto à prevalência de problemas de externalização e internalização. Neste sentido, Rutter et al. (2001), por exemplo, verificaram que crianças que foram adotadas internacionalmente não evidenciavam uma maior incidência daqueles problemas, quando comparadas com crianças com adoção nacional e integradas em famílias do Reino Unido mais precocemente. Por sua vez, noutro estudo também não foram identificadas diferenças significativas, ao nível dos problemas de externalização e internalização, entre um grupo de crianças que foram adotadas e um grupo de crianças da comunidade (Palacios \& Sánchez-Sandoval, 2005).

Perante a divergência entre estudos, importa colocar a seguinte questão: as crianças filhas por adoção evidenciam mais problemas de comportamento do que os seus pares da comunidade? Para responder a esta questão, uma série de meta-análises (Juffer \& Van IJzendoorn, 2005) foram levadas a cabo. Os autores identificaram diferenças entre as crianças que foram adotadas e as da comunidade, quer ao nível dos problemas de externalização, quer ao nível dos problemas de internalização, com resultados mais positivos a favor dos pares da comunidade. Ainda assim, o tamanho do efeito era pequeno ( $d=-.24$ e $d=-.16$, respetivamente). Uma diferença substancial residia entre os dois grupos: as crianças que foram adotadas estavam sobrerepresentadas nos serviços de saúde mental, devido sobretudo à presença de dificuldades de aprendizagem. 


\section{Conclusão}

Os resultados dos estudos mencionados ao longo deste artigo apontam para a ocorrência de mudanças positivas no desenvolvimento da criança após a sua integração numa família por adoção. De acordo com o modelo proposto por Van IJzendoorn e Juffer (2006), a mudança mais rápida e acentuada tenderá a ocorrer no desenvolvimento físico, em particular no peso e no comprimento, seguindo-se o desenvolvimento cognitivo e a segurança da vinculação. Por outro lado, esse modelo adianta, em consonância com resultados de estudos mencionados neste artigo, que anos após a adoção tendem a persistir dificuldades, nomeadamente problemas de comportamento. Este não é o único domínio no qual a investigação identifica défices a longo prazo. Rutter et al. (2001) falam da Síndrome de Pós-Institucionalização, ao salientarem a manutenção de problemáticas particulares anos após a adoção, entre elas comportamentos atípicos (e.g., comportamentos estereotipados, características autisticas), défice de atenção e comportamento indiscriminado de vinculação.

\section{Heterogeneidade nos Resultados Desenvolvimentais}

Apesar dos ganhos observados em diferentes domínios do desenvolvimento, os investigadores têm vindo a salientar, simultaneamente, a heterogeneidade encontrada nos resultados (e.g., Pormerleau et al., 2005). Em particular, os estudos sobre o impacto da adoção no desenvolvimento da criança têm analisado o contributo exercido pelas seguintes variáveis: tempo de institucionalização e o tempo de permanência na família por adoção. Contudo, a investigação tem vindo a apontar para a influência de outras variáveis possivelmente envolvidas nos mecanismos explicativos da mudança e da variabilidade observada após a adoção. Tal como salientado por Rutter et al. (2009), um entendimento daquela heterogeneidade deverá estar alicerçada na combinação de três fatores, nomeadamente (a) as experiências pré-natais, (b) as de cuidados anteriores e posteriores à adoção, e (c) a constituição genética.

Palacios e Brodzinsky (2010), assentes numa perspetiva histórica, apontam para a necessidade da investigação atual sobre a temática estar centrada na identificação de fatores neurobiológicos, desenvolvimentais e relacionais possivelmente envolvidos na experiência da adoção. Desta forma, é fundamental o estudo da interação entre as características da criança e as características dos contextos nos quais aquela se movimenta, tanto antes como depois da adoção. Assim sendo, realçamos o contributo (a) de características atribuídas à criança (e.g., fatores genéticos, temperamento), (b) de características atribuídas aos cuidados prestados na família biológica (e.g., experiências de maus-tratos) e na instituição (e.g., responsividade e indivi- 
dualização dos cuidados), e (c) de características atribuídas ao contexto da família por adoção (e.g., sensibilidade materna, modelos internos dinâmicos e função reflexiva dos pais por adoção).

Quanto a esta última dimensão parecem ser de particular relevância os resultados de programas de intervenção conduzidos com famílias por adoção e de acolhimento (e.g., Circle of Security, Attachment and Biobehavioral Catch-up), que têm vindo a demonstrar o impacto positivo dos comportamentos sensíveis maternos na segurança e organização da vinculação do/a filho/a por adoção (Dozier, Lindhiem, \& Ackerman, 2005), bem como na promoção de um desenvolvimento cognitivo e social adaptado (e.g., Jaffari-Bimmel, Juffer, Van IJzendoorn, Bakermans-Kranenburg, \& Mooijaart, 2006), exercendo uma influência positiva também ao nível neuroendocrino (Dozier et al., 2005). Subjacente aos programas de intervenção mencionados está o pressuposto de que uma relação de vinculação segura entre a criança e a/o mãe/pai adotiva/o constituiu-se como uma dimensão fundamental e um contributo necessário para a construção de uma trajetória de desenvolvimento posterior adaptada.

\section{Contributo para a Investigação Acerca da Adoção Nacional}

Os resultados de estudos que analisaram o impacto da adoção internacional no desenvolvimento da criança podem, assim, revestir-se de particular importância para delinear e refletir acerca de futuras investigações sobre a adoção nacional.

Desta foram, e com base na sistematização realizada e nas suas conclusões, consideramos premente a realização de estudos centrados na monitorização das trajetórias de desenvolvimento de filhos por adoção, iniciando-se no momento de chegada da criança ao contexto institucional, com períodos curtos entre as avaliações. Contrapondo aos frequentes estudos transversais e de carácter retrospetivo, aquele desenho de investigação poderá vir a possibilitar aos investigadores uma análise mais aprofundada do impacto específico no desenvolvimento de experiências anteriores à institucionalização e da própria institucionalização. Aliado a isto, a elaboração de estudos longitudinais, assente em reavaliações posteriores e em diferentes estádios do desenvolvimento, poderá vir ainda a ser um veículo privilegiado para testar se a recuperação no desenvolvimento físico, mental e sócio-emocional da criança está limitada pelo nível de privação vivenciado precocemente. Pela sua escassez e relevância, sublinhamos também a necessidade de realização de mais estudos centrados na compreensão de várias características das famílias por adoção (e.g., variáveis indicadoras da qualidade da relação, práticas educativas parentais, motivações subjacentes à adoção), conforme o domínio desenvolvimental em estudo, assim como na obtenção de informações aprofundadas acerca da família biológica (i.e., fatores genéticos e ambientais). 


\section{Referências}

American Psychiatric Association (2000). Diagnostic and statistical manual of mental disorders ( $4^{\text {th }}$ ed. - Rev. ed.). Washington DC: American Psychiatric Association.

Ames, E. (1997). The development of Romanian orphanage children adopted into Canada. Final report to human resources development, Canada: Burnaby.

Bakwin, H. (1949). Emotional deprivation in infants. Journal of Pediatrics, 35, 512-521. doi:10.1016/S0022-3476(49)80071-0

Bauer, P. M., Hanson, J. L., Pierson, R. K., Davidson, R. J., \& Pollak, S. D. (2009). Cerebellar volume and cognitive functioning in children who experienced early deprivation. Biological Psychiatry, 66, 1100-1106.

Beckett, C., Brendkamp, D., Castle, J., Groothues, C., O’Connor, T., Rutter, M., \& The ERA Study Team. (2002). Behavior patterns associated with institutional deprivation: A study of children adopted from Romania. Developmental and Behavioral Pediatrics, 23(5), 297-303.

Benoit, T.C., Joycelyn, L.J., Moddemann, D.M., \& Embree, J.E. (1996). Romanian adoption. The Manitoba experience. Archives of Pediatrics \& Adolescent Medicine, 150(12), 1278-82.

Bowlby, J. (1951). Maternal care and mental health. Geneva: World Health Organization.

Bowlby, J. (1982). Attachment and loss. Attachment. New York: Basic Books.

Cohen, J. (1988). Statistical power analysis for the behavioural sciences (rev. edn). New York: Academic Press.

Cohen, N., \& Farnia, F. (março, 2011). Children adopted from China: Attachment security two years later. Comunicação apresentada no SRCD Bienal Meeting, Montreal, Canadá.

Cohen, N., Lojkasek, M., Zadeh, Z. Y., Pugliese, M., \& Kiefer, H. (2008). Children adopted from China: A prospective study of their growth and development. Journal of Child Psychology and Psychiatry, 49(4), 458-468. doi: 10.1111/j.1469-7610.2007.01853.x

Dozier, M., Lindhiem, O., \& Ackerman, J. (2005). Attachment and biobehavioral catch-up. In L. Berlin, Y. Ziv, L. Amaya-Jackson, \& M.T. Greenberg (Eds.), Enhancing Early Attachments (pp. 178-195). New York: Guilford.

Frank, D., Klass, P., Earls, F., \& Eisenberg, L. (1996). Infants and young children in orphanages: One view from pediatrics and child psychiatry. Pediatrics, 97(4), 569-578.

Fried, R., \& Mayer, M. F. (1948). Socioemotional factors accounting for growth failure in children living in an institution. Journal of Pediatrics, 33, 444-457. doi: 10.1016/S0022-3476(48)80202-7

Gunnar, M. R. (2000). Early adversity and the development of stress reactivity and regulation. In C. Nelson (Ed.), The effects of early adversity on neurobehavioral development. Minnesota symposia on child psychology (vol. 31, pp. 163200). Mahwah, NJ: Erlbaum. 
Haugaard, J., \& Hazan, C. (2003). Adoption as a natural experiment. Developmental and Psychopathology, 15(4), 909-926. doi:10.1017/S0954579403000427

Jaffari-Bimmel, N., Juffer, F., Van IJzendoorn, M., Bakermans-Kranenburg, M., \& Mooijaart, A. (2006). Social development from infancy to adolescence: Longitudinal and concurrent factors in an adoption sample. Developmental Psychology, 42(6), 1143-1153. doi: 10.1037/0012-1649.42.6.1143

Johnson, D. E. (2000). Medical and developmental sequelae of early childhood institutionalization in Eastern European adoptees. In C. Nelson (Ed.), The effects of early adversity on neurobehavioral development. Minnesota symposia on child psychology (vol. 31, pp. 113-162). Mahwah, NJ: Erlbaum.

Johnson, D. E. (2002). Adoption and the effects on child development. Early human development, 68(1), 39-54.

Juffer, F., Bakermans-Kranenburg, M. J., \& Van IJzendoorn, M. (2005). The importance of parenting in the development of disorganized attachment: Evidence from a preventive intervention study in adoptive families. Journal of Child Psychology and Psychiatry, 46, 263-274. doi: 10.1111/j.1469-7610.2004.00353.x

Juffer, F., \& Rosenboom, L. (1997). Infant-mother attachment of internationally adopted children in the Netherlands. International Journal of Behavioral Development, 20(1), 93-107. doi: 10.1080/016502597385469

Juffer, F., \& Van IJzendoorn, M. (2005). Behavior problems and mental health referrals of international adoptees, JAMA, 293, 2501-2515. doi: 10.1001/jama.293.20.2501

Juffer, F., \& Van IJzendoorn, M. (2009). International adoption comes of age: Development of international adoptees from a longitudinal and meta-analytic perspetive. In G. Wrobel \& E. Neil (Eds), International advances in adoption research and practice (pp. 169-192). Sussex: Wiley-Blacwell.

Kumsta, R., Kreppner, J., Rutter, M., Beckett, C., Castle, J., Stevens, S., \& Sonuga-Barke, E. (2010). Deprivation-specific psychological patterns. Monographs of the Society for Research in Child Development, 75(1), 48-78. doi: 10.1111/j.1540-5834.2010.00550.x.

Le Mare, L. L., \& Audet, K. (2006). A longitudinal study of the physical growth and health of postinstitutionalized Romanian adoptees. Paediatrics \& Child Health, 11(2), 85-91.

Loman, M., Wiik, K., Frenn, K., Pollack, S., \& Gunnar, M. (2009). Postinstitutionalized children's development: Growth, cognitive, and language outcomes. Journal of Development \& Behavioral Pediatrics, 30(5), 426-434. doi: 10.1097/DBP.0b013e3181b1fd08

Londen, M., Juffer, F., \& Van IJzendoorn, M. (2007). Attachment, cognitive, and motor development in adopted children: Short-term outcomes after international adoption. Journal of Pediatric Psychology 32(10), 1249-1258. doi:10.1093/jpepsy/jsm062

Marcovitch, S., Goldberg, S., Gold, A., Washington, J., Wasson, C., Krekewich, K., \& Handley-Derry, M. (1997). Determinants of behavioural problems in Romanian children adopted in Ontario. International Journal of Behavioral Development, 20(1), 17-31. doi:10.1080/016502597385414 
Merz, E., \& Mcall, R. (2010). Parent ratings of executive functioning in children adopted from psychosocially depriving institutions. Journal of Child Psychology and Psychiatry, 52(5), 537-546. doi: 10.1111/j.1469-7610.2010.02335.x

Miller, L., Chan, W., Comfort, K., \& Tirella, L. (2005). Health of children adopted from Guatemala: Comparison of orphanage and foster care. Pediatrics, 115(6), 710-717. doi: 10.1542/peds.2004-2359

Miller, L., \& Hendric, N. (2002). Health of children adopted from China. Pediatrics, 105(6), 1-6. doi: 10.1542/peds.105.6.e76

O'Connor, T. G., Rutter, M., \& The ERA Study Team (2000). Attachment disorder behavior following early severe deprivation: Extension and longitudinal follow-up. Journal of the American Academy of Child and Adolescent Psychiatry, 39, 703-712. doi: 10.1097/00004583-200006000-00008

O’Connor, T. G., \& Spagnola, M. (2009). Early stress exposure: Concepts, findings, and implications, with particular emphasis on attachment disturbances. Child and Adolescent Psychiatry and Mental Health, 3(1), 1-7. doi:10.1186/1753$-2000-3-24$

O’Connor, T. G., \& Zeanah, C. (2003). Introduction to the special issue: Current perspectives on assessment and treatment of attachment disorders. Attachment and Human Development, 5(3), 221-222. doi:10.1080/14616730310001593992

Palacios, J., \& Brodzinsky, D. (2010). Adoption research: Trends, topics, outcomes. International Journal of Behavioral Development, 34(3), 270-284. doi: 10.1177/0165025410362837

Palacios, J., \& Sánchez-Sandoval, Y. (2005). Beyond adopted/nonadopted comparisons. In D. Brodzinsky \& J. Palacios (Eds.), Psychological issues in adoption (pp. 117-144). London: Praeger.

Pollak, S., Nelson, C., Schlaak, M. F., Roeber, B. J., Wewerka, S. S., Wiik, K. L. (...) \& Gunnar, M. (2010). Neurodevelopmental effects of early deprivation in postinstitutionalized children. Child Development, 81, 224-236. doi: 10.1111/j.1467-8624.2009.01391.x

Pormerleau, A., Malcuit, G., Chicoine, J., Séguin, R., Belhumeur, C., Germain, P. (...) \& Jéliu, G. (2005). Health status, cognitive and motor development of young children adopted from China, East Asia, and Russia across the first 6 months after adoption. International Journal of Behavioral Development, 29(5), 445-457. doi: 10.1080/01650250500206257

Provence, S., \& Lipton, R. (1962). Infants reared in institutions. New York: International Universities Press.

Rutter, M., Beckett, C., Castle, J., Colvert, E., Kreppner, J., Mehta, M. (...) \& Sonuga-Barke, E. (2009). Effects of profound early institutional deprivation: An overview of findings from a UK longitudinal study of Romanian adoptees. In G. M. Wrobel \& E. Neil (Eds.), International advances in adoption research for practice (pp. 147-168). Sussex: Wiley-Blackwell.

Rutter. M., Colvert, E., Kreppner, J., Beckett, C., Groothues, C., Hawkins, A. (...) \& Sonuga-Barke, E. (2007). Early adolescent outcomes for institutionally-deprived and non-deprived adoptees. I: Disinhibited attachment. Journal of Child Psychology and Psychiatry, 48(1), 17-30. doi: doi:10.1111/j.1469$-7610.2007 .01792 . x$ 
Rutter, M., Kreppner, J., O’Connor, T. G., \& The ERA Study Team (2001). Specificity and heterogeneity in children's responses to profound privation. British Journal of Psychiatry, 179, 97-103. doi: 10.1192/bjp.179.2.97

Rutter, M, \& The ERA Study Team (1998). Developmental catch-up, and deficit, following adoption after severe global early privation. Journal of Child Psychology and Psychiatry, 39, 465- 476.

Rutter, M., O'Connor, T., \& The ERA Study Team (2004). Are there biological programming effects for psychological development? Findings from a study of Romanian adoptees. Developmental Psychology, 40(1), 81-94. doi: 10.1037/0012-1649.40.1.81

Smyke, A. T., Koga, S., Johnson, D. E., Zeanah, C., \& The BEIP Core Group (2004). The caregiving context in institution-reared and family-reared infants and toddlers in Romania. Journal of Child Psychology and Psychiatry, 48(2), 210-218. doi: 10.1111/j.1469-7610.2006.01694.x

Spitz, R. A. (1945). Hospitalism: An inquiry into the genesis of psychiatric conditions in early childhood. Psychoanalytic Study of the Child, 1, 53-74.

Tan, T., \& Yang, Y. (2005). Language development of Chinese adoptees 18-35 months old. Early Childhood Research Quarterly, 20(1), 57-68. doi: 10.1007/BF02724092

Teilmann, G., Pedersen, C., Skakkebaek, N., \& Jensen, T. K. (2006). Increased risk of precocious puberty in internationally adopted children in Denmark. Pediatrics, 118(2), 391-399. doi: 10.1542/peds.2005-2939

Tuvemo, T., \& Proos, L. A. (1993). Girls adopted from developing countries: A group at risk of early pubertal development and short final height. Implications for health surveillance and treatment. Annals of Medicine, 25(3), 217-219.

Van den Dries, L., Juffer, F., Van IJzendoorn, M., \& Bakermans-Kranenburg, M. (2009). Fostering security? A meta-analysis of attachment in adopted children. Children and Youth Services Review, 31, 410-421. doi:10.1016/j.childyouth.2008.09.008

Van den Dries, L., Juffer, F., Van IJzendoorn, M., \& Bakermans-Kranenburg, M. (2010). Infants' physical and cognitive development after international adoption from foster care or institutions in China. Journal of Developmental \& Behavioral Pediatrics, 31, 144-150. doi: 10.1097/DBP.0b013e3181cdaa3a

Van IJzendoorn, M., Bakermans-Kranenburg, M. J., \& Juffer, F. (2007). Plasticity of growth in height, weight and head circumference: Meta-analytic evidence of massive catch-up after international adoption. Journal of Developmental and Behavioral Pediatrics, 28, 334-343. doi:10.1097/DBP.0b013e31811320aa

Van IJzendoorn, M., \& Juffer, F. (2006). The Emanuel Miller Memorial Lecture 2006: Adoption as intervention. Meta-analytic evidence for massive catch-up and plasticity in physical, socioemotional, and cognitive development. Journal of Child Psychology and Psychiatry, 47(12), 1228-1245. doi:10.1111/j.1469-7610.2006.01675.x

Van IJzendoorn, M., Juffer, F., \& Klein Poelhuis, C. (2005). Adoption and cognitive development: A meta-analytic comparison of adopted and nonadopted children's IQ and school performance. Psychological Bulletin, 131, 301-316. doi:10.1037/0033-2909.131.2.301 
Van IJzendoorn, M., \& Kroonenberg, P. M. (1988). Cross-cultural patterns of attachment: A meta-analysis of the Strange Situation. Child Development, 59, 147-156.

Van IJzendoorn, M., Schuengel, C., \& Bakermans-Kranenburg, M. (1999). Disorganized attachment in early childhood: Meta-analysis of precursors, concomitants, and sequelae. Development and Psychopathology, 11(2), 225-250.

Wallin, A., Dozier, M., Bick, J., \& Bernard, K. (2011, março). Attachment and empathy among children experiencing early adversity. Comunicação apresentada no SRCD Bienal Meeting, Montreal, Canadá.

Wiik, K. L., Loman, M., Van Ryzin, M. J., Armstrong, J. M., Essex, M. J., Pollak, S., \& Gunnar, M. (2011). Behavioral and emotional symptoms of post-institutionalized children in middle childhood. The Journal of Child Psychology and Psychiatry, 52(1), 56-63. doi:10.1111/j.1469-7610.2010.02294.x

World Health Organization (2007). International statistical classification of diseases and related health problems 10th revision (ICD-10). Retirado de http://www.who.int/classifications/icd/en/.

Zeanah, C., Egger, H., Smyke, A., Nelson, C., Fox, N., Marshall, P., \& Guthrie, D. (2009). Institutional rearing and psychiatric disorders in Romanian preschool children. American Journal of Psychiatry, 166, 7 77-785. doi: 10.1176/appi.ajp.2009.08091438

Zeanah, C., Smyke, A., Koga, S., Carlson, E., \& The Beip Core Group (2005). Attachment in institutionalized and community children in Romania. Child Development, 76, 1015-1028. doi:10.1111/j.1467-8624.2005.00894.x 